\section{Articular Involvement in Disseminated Histoplasmosis in a Kidney Transplant Patient Taking Azathioprine}

\section{To the Editor:}

Histoplasmosis can manifest itself in the joints of immunocompromised patients. It is a potentially fatal opportunistic infection and should be considered when these patients present with unexplained joint swelling, erythema nodosum, or sepsis.

An 81-year-old farmer from Minnesota developed acute-onset painful swelling over the ulnar aspect of the right palm. He denied work-related injury or trauma. Hand radiographs did not show evidence of a foreign body. Cephalexin was started for presumed cellulitis, but 4 days later the hand lesion evolved into a localized area of purple discoloration, $3 \times 2.5$ $\mathrm{cm}$, with central clearing. He developed generalized weakness, myalgias, large-joint arthralgias, worsening hand erythema, productive cough, fever $\left(101.2^{\circ} \mathrm{F}\right)$, and hypotension $(88 / 59 \mathrm{~mm} \mathrm{Hg})$, and was hospitalized for presumed sepsis. He was treated empirically with vancomycin and ceftriaxone. Physical examination showed a nontender, nonfluctuant, nonindurated ecchymotic lesion on the ulnar aspect of his right hand distal to the hypothenar eminence, with full active and passive range of motion of wrist and fingers and no tenderness along the digital tendon sheaths. There was no synovitis in the metacarpophalangeal, interphalangeal, or radiocarpal joints. His right knee and ankle were warm and swollen with limited range of motion but no erythema. He had a small palatal ulcer.

His history was pertinent for hypertension, hyperlipidemia, ischemic cardiomyopathy, gout, and degenerative arthritis of the knees. He underwent a cadaveric kidney transplant in 1982 because of endstage renal disease from chronic interstitial nephritis and was maintained on azathioprine $100 \mathrm{mg}$ and prednisone $5 \mathrm{mg}$ daily with excellent allograft function. Surgical history was pertinent for splenectomy in 1981.

Laboratory studies showed hemoglobin $12.1 \mathrm{~g} / \mathrm{dl}$ and white blood cell count $4.8 \times 10^{9} / 1$. Serum creatinine was $1.9 \mathrm{mg} / \mathrm{dl}$, up from a baseline of $1.4 \mathrm{mg} / \mathrm{dl}$. C-reactive protein was $79.4 \mathrm{mg} / \mathrm{l}$ (normal $<8 \mathrm{mg} / \mathrm{l})$. Human immunodeficiency virus serology and BK virus quantitative polymerase chain reaction (PCR) were negative. Blood cultures for aerobic and anaerobic bacteria and fungi were negative after 5 days. Urine cultures were negative. Chest radiograph showed new interstitial opacities with diffuse micronodularity and a miliary pattern. A noncontrast magnetic resonance imaging study of the right hand demonstrated an increased T2 signal abnormality within the soft tissues involving the ulnar aspect of the hand compatible with cellulitis without synovitis, abscess, or osteomyelitis. Radiographs of the right knee showed advanced degenerative changes. Forty milliliters of clear synovial fluid was aspirated from the right knee and revealed 2277 nucleated cells (13\% neutrophils, 24\% lymphocytes, and $63 \%$ monocytes/macrophages) and calcium pyrophosphate crystals, but no urate crystals, malignant cells, or blasts. With negative gram stain, acid-fast bacillus stain, and cultures from the synovial fluid after 48 hours, he was given an intraarticular steroid injection for symptomatic relief of degenerative arthritis and pseudogout.

On hospital day 4, results of Histoplasma capsulatum urine antigen test returned at $6.45 \mathrm{ng} / \mathrm{ml}$ and $H$. capsulatum blood antigen at $116 \mathrm{ng} / \mathrm{ml}$ (normal = undetectable). Fungal immunodiffusion studies showed positive $\mathrm{H}$ and $\mathrm{M}$ bands consistent with disseminated histoplasmosis. H. capsulatum was also identified by DNA probe in the synovial fluid from the right knee. Punch biopsy from the right palm (Figure 1) showed intense lymphohistiocytic infiltrate with numerous deep dermal fungal microorganisms on Grocott's methenamine silver staining consistent with $H$. capsulatum. In situ hybridization studies for $H$. capsulatum were also positive. The patient was started on itraconazole for disseminated histoplasmosis involving the skin, palatal mucosa, joints, and lungs. He gradually showed clinical improvement with resolution of fever, palmar cellulitis, and palatal ulcer. At the 9-month followup he remained symptom-free, with complete resolution of knee and ankle pain and swelling. He continues to take itraconazole in view of chronic immunosuppression for his transplanted kidney.

Histoplasmosis, caused by H. capsulatum (a dimorphic fungus found in bird and bat fecal material), is endemic in the Mississippi and Ohio River valleys and certain parts of Central and South America. Most infections are subclinical and self-limited but can be potentially fatal in immunocompromised hosts if not recognized and treated. Rheumatologic manifestations of histoplasmosis are very uncommon. In a 15-year retrospective review of 111 patients with systemic histoplasmosis from our institution, no cases with joint involvement were documented ${ }^{1}$. Clinical presentation can often mimic septic, crystalline, or degenerative arthritis, as in our patient. Both native and prosthetic joints can be affected. Arthralgias or polyarticular arthritis ${ }^{2}$ with or without erythema nodosum or erythema multiforme may occur as part of primary acute histoplasmosis. Dissemination occurs in $<0.1 \%^{3}$, particularly in the elderly or immunocompromised. Suppurative arthritis may accompany disseminated histoplasmosis or occur as solitary monoarthritis. Osteomyelitis ${ }^{4}$, spondylodiscitis, recurrent tenosynovitis, and carpal tunnel syndrome ${ }^{5}$ have also been described in disseminated histoplasmosis.

Rheumatologic manifestations were noted in 6\% (24 of 381) of patients with histoplasmosis during 1 epidemic $^{6}$. In 3 patients these were the main presenting complaints. There was rapidly additive joint involvement, symmetric in $50 \%$, although 10 had monoarticular or oligoarticular disease. Small joints of the hand, wrists, knees, and ankles were the commonest sites affected, in contrast to the predominantly migratory pattern of large lower extremity joint involvement seen in another epidemic ${ }^{7}$. Erythema nodosum was noted in 11 of these patients, who also exhibited systemic features (fever, anemia, and elevated erythrocyte sedimentation rate). Sixteen $(67 \%)$ sought medical attention and symptoms resolved without treatment or with a brief course of nonsteroidal antiinflammatory drugs in all but 2 patients.

Diagnosis is based on clinical suspicion with detection of histoplasma antigen in urine or serum or successful demonstration of $H$. capsulatum in cultures or histologic specimens by in situ hybridization or $\mathrm{PCR}^{8,9}$. Traditionally, successful treatment has been accomplished with amphotericin $\mathrm{B}^{10}$ in patients with fulminant disease or associated sepsis but triazole antifungals are noted to be as effective. Itraconazole (200 mg twice daily for 12 months) is the treatment of choice for disseminated histoplasmosis with mild or moderately severe symptoms not requiring hospitalization $^{11}$. In immunosuppressed patients, antifungal therapy should be accompanied by a decrease in the dose of immunosuppressant if possible. Rarely, surgical debridement of joints or amputation may be necessary. Urine and serum antigen levels typically fall with effective therapy and can be used to follow treatment response and assess for relapse ${ }^{12}$. Antigen levels should be measured before treatment is initiated, at 2 weeks and 1 month, and then every 3 months during therapy with monitoring continued at least 6 months after therapy is discontinued ${ }^{13}$. Persistent low-level antigenuria is frequently observed in infected organ transplant recipients despite complete clinical response after therapy. Limited experience suggests that antifungal therapy can be safely withdrawn in this situation with careful monitoring for relapse $\mathrm{e}^{14,15}$. Longer treatment regimens in immunocompromised or transplant patients are not supported by any evidence ${ }^{12}$.

Histoplasmosis is a potentially fatal opportunistic infection causing articular manifestations that rheumatologists should keep in mind when patients in endemic areas taking immunosuppression present with unexplained joint swelling, erythema nodosum, or sepsis. Urine and serum histoplasma antigen testing should be sought for screening as they allow for a quicker diagnosis than cultures and are more readily available than PCR or in situ hybridization testing. DNA probe for $H$. capsulatum or fungal culture can help identify the infection in synovial fluid. Success of therapy and identification of relapse can also be monitored over time by antigen testing.

ASHIMA MAKOL, MD, Division of Rheumatology, Department of Internal Medicine; CARILYN N. WIELAND, MD, Department of Dermatology; STEVEN R. YTTERBERG, MD, Division of Rheumatology, Department of Internal Medicine, Mayo Clinic, Rochester, Minnesota, USA. Address correspondence to Dr. A. Makol, Mayo Clinic, Division of Rheumatology, Department of Internal Medicine, 200 First St. SW, Rochester, MN 55905, USA. E-mail: makol.ashima@mayo.edu 

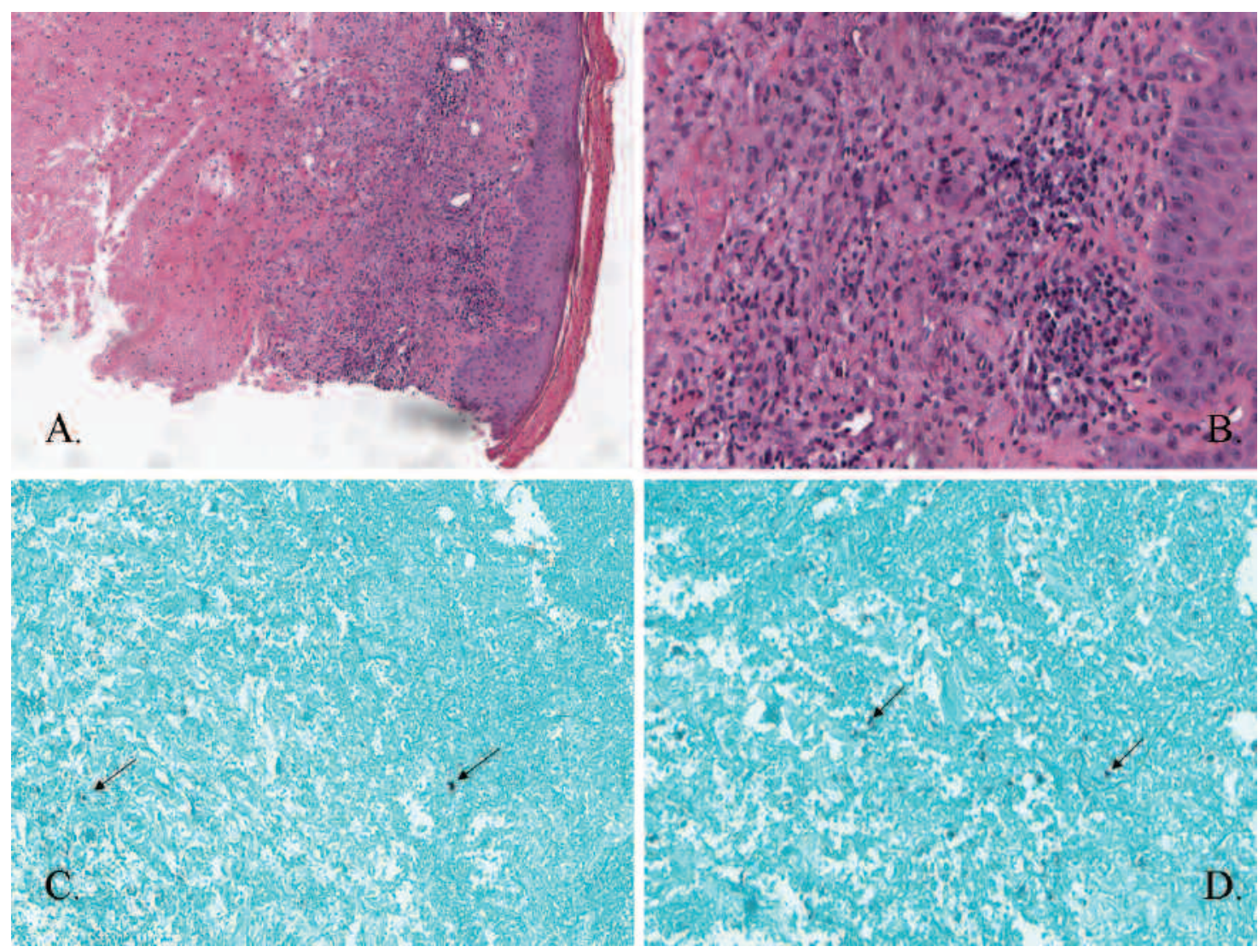

Figure 1. Right palm skin biopsy: panels A $(\times 6)$ and B $(\times 20)$, H\&E staining, demonstrate dense granulomatous inflammation throughout the dermis with abundant histiocytes. No infectious organisms were identified. Panels $\mathrm{C}(\times 10)$ and $\mathrm{D}(\times 20)$, Grocott's methenamine silver staining, demonstrate numerous yeast-like structures throughout the mid- and deep dermis (arrows). Some had surrounding pseudocapsules and demonstrated narrow-based budding. These deep dermal fungal microorganisms were morphologically consistent with $H$. capsulatum. Panfungal and histoplasmosis in situ hybridization studies were positive. Gram and Fite stains were negative.

\section{REFERENCES}

1. Assi MA, Sandid MS, Baddour LM, Roberts GD, Walker RC. Systemic histoplasmosis: A 15-year retrospective institutional review of 111 patients. Medicine 2007;86:162-9.

2. Weinberg JM, Ali R, Badve S, Pelker RR. Musculoskeletal histoplasmosis. A case report and review of the literature. J Bone Joint Surg Am 2001;83:1718-22.

3. Cuellar ML, Silveira LH, Citera G, Cabrera GE, Valle R. Other fungal arthritides. Rheum Dis Clin North Am 1993;19:439-55.

4. Kohli R, Hadley S. Fungal arthritis and osteomyelitis. Infect Dis Clin North Am 2005;19:831-51.

5. Cucurull E, Sarwar H, Williams CS 4th, Espinoza LR. Localized tenosynovitis caused by Histoplasma capsulatum: Case report and review of the literature. Arthritis Rheum 2005;53:129-32.

6. Rosenthal J, Brandt KD, Wheat LJ, Slama TG. Rheumatologic manifestations of histoplasmosis in the recent Indianapolis epidemic. Arthritis Rheum 1983;26:1065-70.

7. Sellers TF Jr, Price WN Jr, Newberry WM Jr. An epidemic of erythema multiforme and erythema nodosum caused by histoplasmosis. Ann Intern Med 1965;62:1244-62.

8. Abbott JJ, Hamacher KL, Ahmed I. In situ hybridization in cutaneous deep fungal infections: A valuable diagnostic adjunct to fungal morphology and tissue cultures. J Cutan Pathol 2006;33:426-32.
9. Kauffman CA. Histoplasmosis: A clinical and laboratory update. Clin Microbiol Rev 2007;20:115-32.

10. Poveda F, Garcia-Alegria J, de las Nieves MA, Villar E, Montiel N, del Arco A. Disseminated histoplasmosis successfully treated with liposomal amphotericin B following azathioprine therapy in a patient from a nonendemic area. Eur J Clin Microbiol Infect Dis 1998; 17:357-9.

11. Wheat J, Sarosi G, McKinsey D, Hamill R, Bradsher R, Johnson P, et al. Practice guidelines for the management of patients with histoplasmosis. Infectious Diseases Society of America. Clin Infect Dis 2000;30:688-95.

12. Proia L, Miller R. Endemic fungal infections in solid organ transplant recipients. Am J Transplant 2009;9 Suppl 4:S199-207.

13. Wheat LJ. Improvements in diagnosis of histoplasmosis. Expert Opin Biol Ther 2006;6:1207-21.

14. Freifeld AG, Iwen PC, Lesiak BL, Gilroy RK, Stevens RB, Kalil AC. Histoplasmosis in solid organ transplant recipients at a large Midwestern university transplant center. Transpl Infect Dis 2005;7:109-15.

15. Wheat LJ, Freifeld AG, Kleiman MB, Baddley JW, McKinsey DS, Loyd JE, et al. Clinical practice guidelines for the management of patients with histoplasmosis: 2007 update by the Infectious Diseases Society of America. Clin Infect Dis 2007;45:807-25.

J Rheumatol 2011;38:12; doi:10.3899/jrheum.110776 